\title{
Dialogia do Conhecimento Pedagógico dos Recursos Tecnológicos e Demais Saberes Docentes
}

\author{
Rosária H. Ruiz Nakashima ${ }^{1}$, Stela C. Bertholo Piconez ${ }^{2}$ \\ ${ }^{1}$ Curso de Licenciatura em História - Universidade Federal do Tocantins (UFT) \\ Av. Paraguai esq c/Rua Uxiramas, s/nº CEP: 77824-838 - Araguaína, TO - Brazil \\ ${ }^{2}$ Faculdade de Educação - Universidade de São Paulo (USP) \\ São Paulo, Brazil \\ rosaria@uft.edu.br, stela.piconez@gmail.com
}

\begin{abstract}
This article presents reflections on the use of Digital Information and Communication Technology from the theoretical perspective substantiated by Technological Pedagogical Content Knowledge (TPACK) in a course on Higher Education in blended learning mode. The data collected and analyzed confirm the existence of a pedagogical knowledge of technological resources that known and articulated to other knowledge, recognize educational intentionality to new ways of learning and teaching, supported by technologies.
\end{abstract}

Resumo. Este artigo apresenta reflexões sobre o uso das tecnologias digitais de informação e comunicação, sob a perspectiva teórica fundamentada pelo Technological Pedagogical Content Knowledge (TPACK), no ensino superior, na modalidade blended learning. Os dados coletados e analisados confirmam a existência de um conhecimento pedagógico dos recursos tecnológicos que, articulados aos demais saberes docentes, podem conferir intencionalidade educativa ao processo de aprendizagem e maior sentido ao processo de avaliação do estudante. Sua relação dialógica agrega valores aos novos modos de aprender e de ensinar, apoiados por tecnologias.

\section{Introdução}

A presença das tecnologias digitais de informação e comunicação (TDIC) tem sugerido transformações na dinâmica docente e nas propostas educacionais, o que requer uma relação dialógica que se estrutura pela existência de diferentes tipos de conhecimentos (conceituais específicos, tecnológicos e pedagógicos). O uso da web (rede de informações), por meio da internet (rede de computadores), tem ampliado os desafios na educação, gerando certa insegurança em professores no processo de seleção e utilização das TDIC [Figueiredo, Nobre e Passos 2015], dada a diversidade de recursos tecnológicos disponíveis. Portanto, o objetivo deste artigo é apresentar, a partir de revisão bibliográfica e de uma experiência didática no ensino superior, perspectivas teóricas e estratégias sobre a formação de educadores para o uso das TDIC.

Muitos autores têm reconhecido a relevância da dialogia entre os diferentes saberes docentes [Mishra e Koehler 2006; Ronau, Rakes e Niess 2012] ao reconhecerem as ideias 
anteriormente defendidas por Shulman $(1986$; 1987) sobre a inter-relação dos diferentes saberes docentes, complementada pelo conhecimento pedagógico dos conteúdos das tecnologias.

A partir da certeza da importância do papel do professor, focalizada em relatórios internacionais [Unesco 2008; Wilson et al. 2011], muitos questionamentos podem ser feitos, tais como: o que é necessário saber ensinar e aprender? Como selecionar recursos tecnológicos que qualifiquem a avaliação da aprendizagem dos estudantes, em termos de desenvolvimento de competências tecnológicas exigidas pela sociedade atual?

O campo de pesquisa sobre saberes docentes vem crescendo, nas últimas duas décadas, na realidade nacional e internacional [Shulman 1987; Schön 1992; Mizukami 2004; Tardif 2004; Nakashima 2014]. Entretanto, é preciso questionar ainda quais são os saberes tecnológicos necessários ao ensino. Eles fazem parte dos diversos tipos de saberes presentes na contemporaneidade: são conhecimentos disciplinares, curriculares, pedagógicos e críticocontextuais. Estão presentes na multidimensionalidade educativa e não podem ser ignorados por serem de grande potencialidade para o trabalho docente e aprendizagem dos estudantes [Mishra e Koehler 2006; Harris 2008; Niess 2008, 2012; Bull, Bell e Hammond 2008; Ronau e Rakes 2012a; Hammond, Alexander e Bodzin 2012; Pape et al. 2012].

As TDIC possuem conteúdo tecnológico (letramento digital, informacional e midiático, usabilidade técnica e pedagógica dos recursos digitais etc.) que incentiva e estimula os estudantes como apoio de suas autoaprendizagens e, também, na direção de outras modalidades de educação, tais como aberta e não formal (educação a distância, $e$ learning, b-learning, u-learning, mobile learning ou com o uso de plataformas virtuais de aprendizagem e de seus aplicativos diversificados).

$\mathrm{Na}$ seção 2 deste artigo será apresentado o modelo explicativo da ação docente, fundamentado nos estudos de Mishra e Khoeler (2006), com descrição da posição do conhecimento pedagógico das tecnologias, na dialogia com os demais saberes docentes. $\mathrm{Na}$ seção 3 serão descritos os procedimentos e estratégias do movimento metodológico que possibilitou as reflexões inseridas neste artigo. Na seção 4 serão discutidos os resultados das reflexões de ordem teórico-práticas desenvolvidas, culminando com as conclusões.

\section{A dialogia dos saberes docentes segundo o modelo TPACK}

As ideias de Shulman (1987) sobre o Pedagogical Content Knowledge (PCK), ou Conhecimento Pedagógico de Conteúdo, representa a intersecção dos conhecimentos de conteúdo específico e pedagógico (Figura 1) que permite a compreensão de como determinados temas se organizam e são adaptados aos diferentes interesses e habilidades dos estudantes. Esse conhecimento não é apenas um repertório de técnicas que o professor utiliza para ensinar um conteúdo, mas se caracteriza por uma forma de pensar, própria do professor, uma habilidade de tornar um conteúdo compreensível para o estudante, considerando os propósitos de ensino.

De acordo com Koehler, Mishra e Yahya (2007), discussões na literatura relatam a extensão do conceito $P C K$, defendido por Shulman, agora, para fundamentar o uso das TDIC. Nesse sentido, o TPACK não é uma abordagem completamente nova e o diferencial se concentra na intersecção dos componentes no modelo que pode auxiliar na análise das mudanças atitudinais, procedimentais e conceituais das competências docentes. 
V Congresso Brasileiro de Informática na Educação (CBIE 2016)

Anais do XXII Workshop de Informática na Escola (WIE 2016)

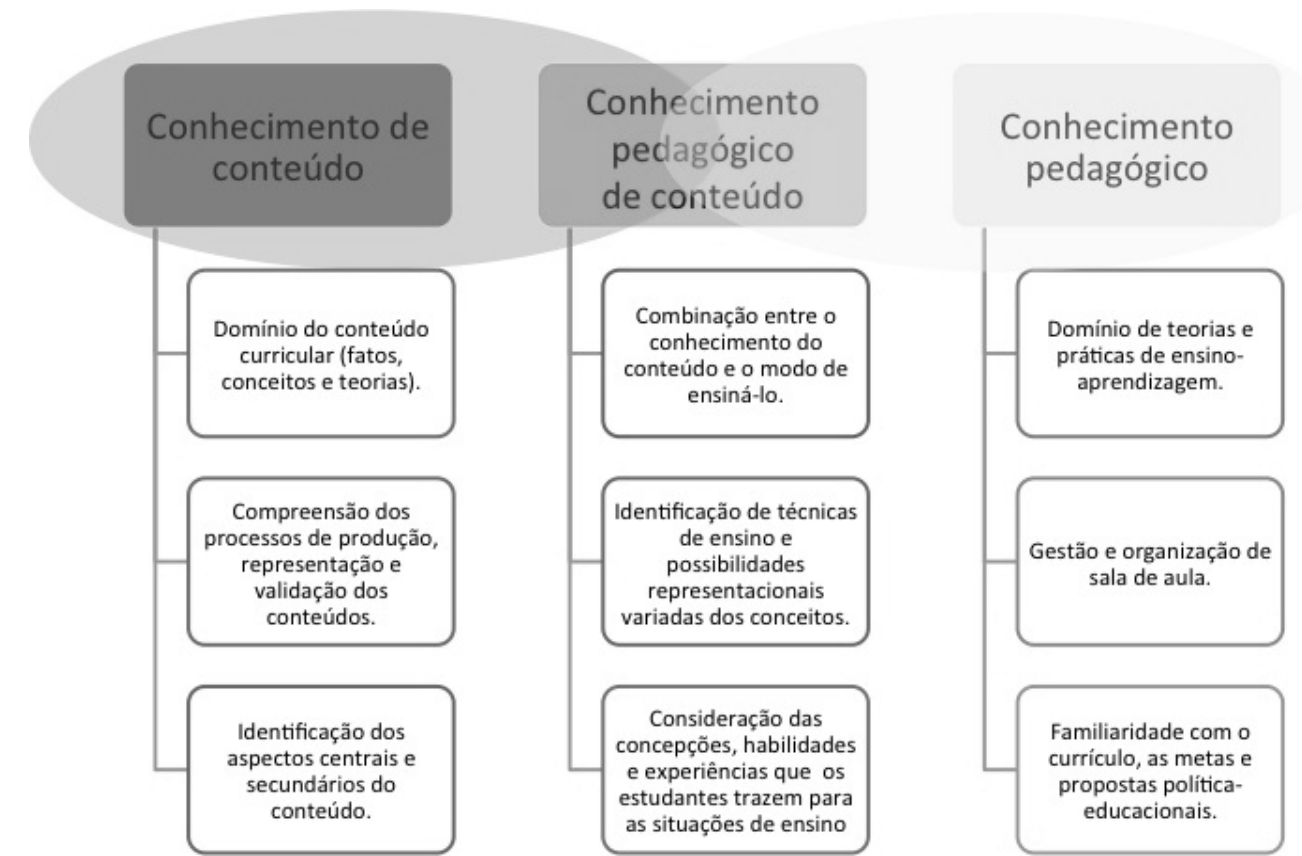

Figura 1- Intersecção dos conhecimentos de conteúdo e pedagógicos, organizado pelas autoras

Segundo Cox e Graham (2008) e Koehler, Shin e Mishra (2012) a estrutura do $T P A C K$, considerada uma complexa interação dos três corpos de conhecimentos, impacta significativamente em pesquisas e práticas de produção de conhecimentos flexíveis, necessários para integrar a tecnologia em sala de aula. As funções do modelo, como um esquema de classificação, fornecem informações sobre a natureza e as relações dos objetos, ideias e ações pedagógicas em interação com as tecnologias selecionadas. A Figura 2 apresenta as diferentes dimensões dos conhecimentos docentes, frente à inserção do conhecimento pedagógico dos conteúdos tecnológicos.

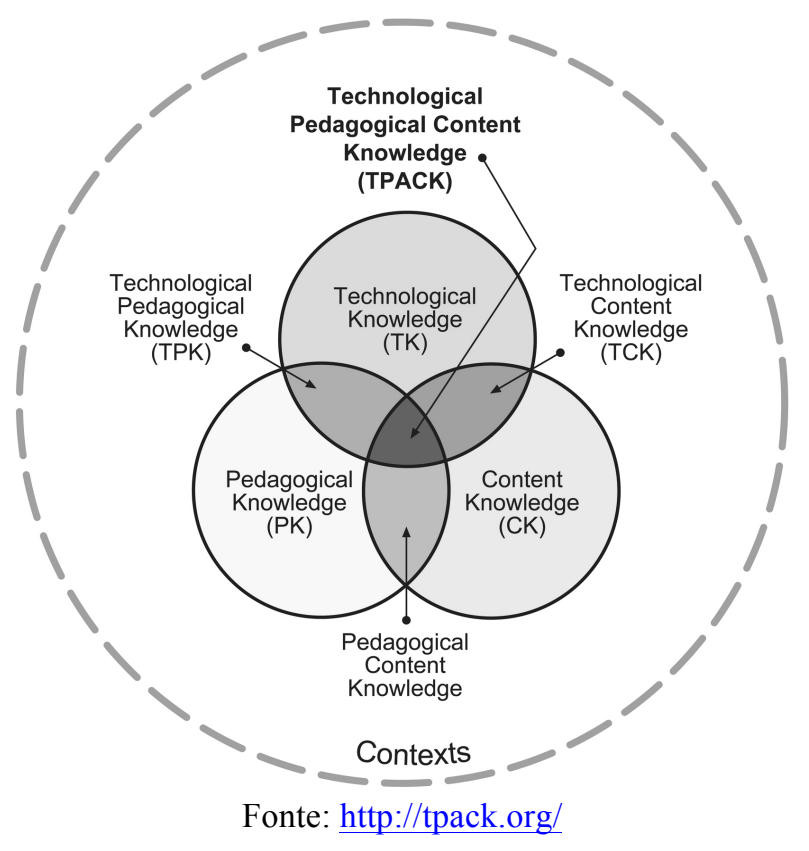

Figura 2 - Framework TPACK e a integração dos seus componentes 
Conforme a Figura 2, o componente Pedagogical Knowledge (PK) concentra-se no conhecimento docente dos domínios de suas abordagens (aprendizagem por descoberta, aprendizagem cooperativa, aprendizagem baseada em problemas, dentre outros. O Content Knowledge (CK) indica o conhecimento das possíveis representações de tópicos específicos em uma determinada área, por exemplo, modelos de fluxos de elétrons em Ciências, gráficos e tabelas em Matemática ou linhas do tempo em História. É um conhecimento que independe de atividades pedagógicas específicas ou de como o professor pode usar essas representações para ensinar. O Technological Knowledge (TK) é definido como o conhecimento sobre o uso específico das tecnologias emergentes. Koehler e Mishra (2008) ainda ressaltam que a tecnologia tem um conteúdo que envolve o conhecimento do histórico da tecnologia (concepção do produto; lançamento no mercado; adoção pelos usuários; atualização das versões e obsolescência técnica ou funcional); das características da tecnologia (o que é; para que serve e como funciona); e das formas encontradas por professores e estudantes para usarem os recursos tecnológicos com intencionalidade educativa.

Na Figura 2, o Conhecimento do Conteúdo Tecnológico (TCK) permite compreender que o design da tecnologia pode contribuir ou restringir o que se pode fazer com ela, bem como compreender a relação das TDIC com o conteúdo curricular específico. Por exemplo, o uso do e-mail contribui para comunicação assíncrona, facilita o armazenamento de arquivos e a trocas de mensagens. Entretanto, dificulta a comunicação síncrona, a expressão de sutilezas, emoção e humor [Koehler e Mishra 2008].

A intersecção (Figura 2) referente ao Conhecimento Pedagógico do Conteúdo Tecnológico $(T P K)$ abrange a compreensão de componentes e potencialidades de várias tecnologias; como elas são usadas no processo de ensino e aprendizagem e de que forma o ensino pode mudar como resultado do uso de uma tecnologia específica [Mishra e Koehler 2006]. Implica em conhecer os diferentes recursos tecnológicos e escolher o mais apropriado para a realização de atividades específicas; dominar estratégias pedagógicas e ter habilidades para aplicá-las com o uso da tecnologia.

Finalmente, a integração dos três componentes (TPACK) se refere ao conhecimento de como coordenar o uso de atividades para disciplinas específicas ou para representar tópicos específicos, utilizando as TDIC para facilitar aprendizagem e subsidiar critérios de avaliação do estudante. Para Mishra e Koehler (2006), o modelo TPACK auxilia no desenvolvimento de ambientes de aprendizagem; prioriza o ensino de habilidades em tecnologias de forma contextualizada e estimula a análise do conhecimento do professor e sua evolução.

\section{O movimento metodológico do estudo}

As reflexões que compõem este artigo fazem parte de uma investigação teórica e empírica à semelhança da reflexão na ação, incluindo aspectos de racionalidade, interpretação e crítica tal como sugerida pelas ideias de Schön (1992) e pelo processo de raciocínio e ação pedagógica defendido por Shulman (1987), agora, com conhecimentos tangíveis para resolver os desafios de ensinar com tecnologias.

O trabalho reflete as análises realizadas em uma pesquisa de natureza qualitativa descritivo-exploratória, com abordagem participante, com objetivo de investigar os componentes basilares do TPACK, presentes na proposta didática de uma disciplina presencial, apoiada pelo Moodle, durante seis meses, no ensino superior. A maioria dos participantes da pesquisa (73\%) tinha formação na área de humanas (Pedagogia, Letras, História e Comunicação), 20\% em exatas (Matemática, Ciência da Computação, Ciências 
Contábeis e Engenharia) e 7\% em biológicas (Enfermagem e Fisioterapia). A procura pela disciplina foi justificada pelos participantes pelo fato de que $54 \%$ atuavam no magistério e buscavam subsídios para o exercício da docência, apoiado por tecnologias.

A disciplina possui um design instrucional e um learning design [Piconez e Filatro 2009] apoiado um ambiente virtual (Moodle) e centraliza estudos e reflexões derivadas das intencionalidades educativas, atribuídas às tecnologias em seu planejamento e seleção.

Sob a perspectiva das reflexões teóricas, foi realizada revisão bibliográfica, sobre a temática, em bases de dados nacionais e internacionais. De um total das 48 produções acadêmicas, 24\% destacaram a integração de conhecimentos pedagógicos e tecnológicos, relacionados à formação geral de professores. Cabe destacar 39 artigos encontrados em dois periódicos (Computers \& Education e British Journal of Educational Technology), com maior fator de impacto em 2010/2011, específicos sobre educação e tecnologia. Desse total, consonantes à temática, $32 \%$ discutiram o conhecimento pedagógico das tecnologias como extremamente relevante para a formação do professor [Tondeur et al 2012; Peeraer; Van Petegem 2012; Petko 2012; Yurdakul et al. 2012; Prestridge 2012; Graham 2011; Archambault e Barnett 2010; Angeli e Valanides 2009; Kilbourn e Álvarez 2008; Koehler, Mishra e Yahya 2007]. Ainda desse total, as produções de Kinchin (2012); Liu (2008); Davis, Preston e Sahin (2009); Ferdig (2006) destacaram a integração dos diferentes saberes, incluindo o conhecimento pedagógico dos conteúdos tecnológicos.

A revisão de estudos também incluiu dez capítulos publicados em dois livros que discutem a inter-relação dos diferentes tipos de conhecimentos na formação geral de professores, apoiada por TDIC, cuja publicação de 2008 foi organizada pela American Association of Colleges for Teacher Education (AACTE) e a de 2012 organizada por Ronau, Rakes e Niess.

Nos Anais do Workshop de Informática na Escola (WIE) foram encontrados apenas dois artigos sobre o modelo TPACK. Harriman e Branch, (2012) exploraram o papel do modelo em um curso de integração tecnológica, voltada para professores em atuação. Já Nicolau, Araújo e Silva (2015) articularam o modelo a uma reflexão sobre desafios e possibilidades da educação a distância, a partir da experiência de gestão de cursos on-line em três diferentes plataformas de ensino a distância.

Concomitante aos procedimentos da revisão teórica, a pesquisa foi acompanhada de observação direta, entrevistas, atividades netnográficas (acompanhamento das atividades nos recursos tecnológicos do Moodle), filmagens, formulários de fluência digital e de avaliação do curso, grupos focais de autoavaliação dos estudantes, diários de campo, utilizados na triangulação de dados.

Esse foi o cenário metodológico que possibilitou destacar as reflexões e contribuições que consideram a proposta de Shulman $(1986,1987)$ e a continuidade dada por Mishra e Koehler (2006) e demais autores citados, vivenciados na referida disciplina de ensino superior. Os resultados encontrados, descritos na próxima seção, são fruto das análises que permitiram identificar os conhecimentos pedagógicos relevantes para atribuição de intencionalidade educativa aos recursos do Moodle. As contribuições explicitadas do modelo $T P A C K$ fundamentaram as reflexões sobre os conhecimentos pedagógicos das tecnologias.

\section{Implicações pedagógicas: alguns resultados}

O conhecimento pedagógico do conteúdo, presente no uso educacional dos recursos tecnológicos, estimulou reflexões entre professor e estudantes sobre a interação necessária 
dos diferentes saberes. De acordo com a seleção e estudo de cada recurso tecnológico (e-mail, chat, fórum, wiki, formulários, glossário, CmapTools, Movie Maker, banco colaborativo de tecnologias e produção individual e colaborativa) foram realizadas reflexões sobre as funcionalidades $\mathrm{e}$ as potencialidades para o processo de aprendizagem. Dentre as competências, tanto do ensino quanto do desenvolvimento da aprendizagem, foram observadas habilidades, tais como capacidade de ampliar a argumentação, criação de conteúdos digitais, elaborados individualmente ou em grupo, estímulo às habilidades de trabalho colaborativo, expansão de conhecimentos pedagógicos (abordagem individualizada e/ou contextualizada; concepção inovadora de avaliação e possibilidades de atividades de recuperação permanente; aprendizagem ativa etc.) e compreensão das competências inter, intra e transdisciplinares, na construção de conhecimentos das tecnologias.

A disciplina favoreceu a meta-análise sobre o uso dos recursos tecnológicos do Moodle, sob perspectiva de ação-reflexão-ação, conferindo sentido e significado em relação à seleção, uso e avaliação da intencionalidade educativa mais adequada ao contexto da disciplina. A partir dessas estratégias, sob perspectiva de learning by doing, os estudantes refletiram sobre o uso do Moodle, utilizando seus recursos para compreender seu potencial pedagógico.

De acordo com a avaliação da disciplina, realizada pelos participantes, alguns excertos ilustraram a perspectiva em questão. O participante A afirmou que "a dinâmica das atividades do curso foi muito bem planejada, que juntamente com as excelentes provocações cognitivas da professora motivaram a vontade de aprender e a participação dos alunos no curso para o desenvolvimento de novas perspectivas educacionais, em relação aos recursos que utilizamos no ambiente virtual". O participante F destacou que "a participação no fórum contribuiu para que eu pudesse experienciar outras possibilidades pedagógicas além das tradicionais como por exemplo sanar dúvidas, responder apenas a perguntas relacionadas a um determinado texto e melhorar minha capacidade de argumentação. Tive a oportunidade de comparar a minha resposta com os argumentos de meus colegas". O participante $\mathrm{E}$ considerou que "nesse tipo de curso, a concepção de avaliação sobre meu desempenho é diferente. Com os recursos tecnológicos as aprendizagens de todos extrapolam a sala de aula e ficam disponíveis nos registros do chat, fórum e wiki. A professora não se limitou a resolver tarefas, mas nos provocou cognitivamente para o estudo e pesquisa das tecnologias mais adequadas quando precisamos representar o conhecimento de diferentes maneiras".

O design pedagógico, orientado pelos resultados das sondagens diagnósticas de fluência digital; conhecimento dos múltiplos aplicativos e compreensão da hipertextualidade, presente no uso das tecnologias, orientou o docente, quanto à intencionalidade educativa dos mesmos, bem como à priorização do desenvolvimento de competências de letramento digital, avaliada positivamente pelos estudantes.

O modelo explicativo da ação docente, fundamentado pelo TPACK, tornou-se campo fértil para reflexões sobre a riqueza de abordagens interdisciplinares, quando as propostas são apoiadas por recursos tecnológicos. Tal fato rebate dados de pesquisas que evidenciam a qualidade de um curso pela quantidade/diversidade de seus recursos tecnológicos. A estrutura analítica do TPACK colaborou com a reflexão docente e discente, e incidiu sobre a organização das competências didático-pedagógicas, ao permitir constantemente o levantamento de alternativas satisfatórias, tanto na organização sequencial da proposta do curso, quanto na seleção adequada das TDIC para atendimento das demandas dos estudantes. Estas podem ser customizadas, considerando os diferentes estilos de aprendizagem, com o objetivo de inovar e complementar novas formas de ensinar e aprender conteúdos específicos. 
V Congresso Brasileiro de Informática na Educação (CBIE 2016)

Anais do XXII Workshop de Informática na Escola (WIE 2016)

\section{Conclusões}

Este artigo apresenta reflexões sobre o uso das TDIC, sob a perspectiva teórica fundamentada pelo TPACK, no ensino superior, na modalidade blended learning. Os dados coletados e analisados confirmam a existência de um conhecimento pedagógico dos recursos tecnológicos que, articulado aos demais saberes docentes, pode conferir intencionalidade educativa ao processo de aprendizagem e maior sentido ao processo de avaliação do estudante. Procura elucidar as estruturas que compõem o quadro teórico do $T P A C K$ e suas contribuições na formação de professores, o que implica um conjunto de conhecimentos e atitudes necessário aos planejamentos educacionais.

Foi identificada na literatura a relevância do entendimento de professores acerca das mudanças provocadas pelas tecnologias na aprendizagem e na vida dos estudantes, incluindo a forma como eles interagem com os recursos tecnológicos, que é diferente de como os professores utilizam os mesmos. De acordo com os estudos revisados, para que os recursos tecnológicos se tornem favoráveis em suas práticas pedagógicas, os professores devem compreender como os estudantes aprendem e o que significa ensinar e aprender com tecnologia. Os estudos destacaram a relevância da integração dos diferentes saberes, incluindo o conhecimento pedagógico dos conteúdos tecnológicos. Uma das características importantes observadas foi que o TPACK pode fundamentar perspectivas de formação inicial e continuada de professores com o objetivo de promover uma efetiva utilização das TDIC em contexto de sala de aula, seja sob a perspectiva presencial, semipresencial ou on-line. Portanto, os professores, ao se prepararem para explorar de maneira significativa as possibilidades atuais de uso das tecnologias podem desenvolver, pelas reflexões sobre o modelo TPACK descrito, atitudes e posturas mais profissionais com a interação e/ou entrelaçamento complexo dos saberes necessários para ensinar e aprender.

O modelo explicativo da ação docente TPACK envolve a especificidade epistemológica da construção do conhecimento em si (saberes pedagógicos, saberes específicos e saberes tecnológicos), das demandas dos estudantes (suas dificuldades e estilos de aprendizagem) e do contexto (social, político, econômico e cultural). Contribui para a compreensão de como determinados temas que são difíceis de serem entendidos pelos estudantes ou difíceis de serem representados por professores, podem ser transformados e ensinados com o apoio das tecnologias.

\section{Referências}

Angeli, Charoula e Valanides, Nicos (2009). Epistemological and methodological issues for the conceptualization, development, and assessment of ICT-TPCK: advances in technological pedagogical content knowledge (TPCK). In Computers \& Education, v. 52, n. 1, p.154-168. Publishing Digital.

Archambault, Leanna M. e Barnett, Joshua H. (2010). Revisiting technological pedagogical content knowledge: Exploring the TPACK framework. In Computers \& Education, v. 55, n. 4, p. 1656-1662. Publishing Digital.

Bull, Glen; Bell, Lynn; Hammond, Tom. (2008) “Advancing TPACK trough collaborations across educational associations”. In: AACTE Committee on Innovation and Technology. Handbook of Technological Pedagogical Content Knowledge for educators. New York: Routledge. Cap. 13, p. 273-287.

Cox, Suzy; Graham, Charles. R. (2009) Diagramming TPCK in Practice: Using and elaborated model of the TPCK framework to analyze and depict teacher knowledge. In TechTrends, v. 53, n. 5, p. 60-69. Publishing Digital. 
V Congresso Brasileiro de Informática na Educação (CBIE 2016)

Anais do XXII Workshop de Informática na Escola (WIE 2016)

Davis, Niki; Preston, Christina; Sahin, Ismail (2009) Training teachers to use new technologies impacts multiple ecologies: Evidence from a national initiative. In British Journal of Educational Technology. V. 40, n. 5, p. 861-878. Publishing Digital.

Figueiredo, Gislaine e Passos Isaura Nobre, Marize Lyra S. (2015). "Tecnologias Computacionais na Educação: Desafios na Prática Docente”. IV Congresso Brasileiro de Informática na Educação (CBIE 2015) e XXI Workshop de Informática na Escola 2015. Disponível em: http://br-ie.org/pub/index.php/wie/article/view/5006/3416. Acesso em 28 mai. 2016.

Ferdig, Richard (2006). Assessing technologies for teaching and learning: understanding the importance of technological pedagogical content knowledge. In British Journal Educational Technology. v. 37, n. 5, p. 749-760. Publishing Digital.

Graham, Charles R. (2011). Theoretical considerations for understanding technological pedagogical content knowledge (TPACK). In Computers \& Education, v. 57, n. 3, p. 1953-1960. Publishing Digital.

Hammond, Thomas C.; Alexander, Curby; Bodzin, Alec M. (2012) "Assessment in Authentic Environments: Designing Instruments and Reporting Results from Classroom-Based TPACK Research”. In: Ronau, Robert N.; Rakes, Christopher R.; Niess Margaret L. Educational Technology, Teacher Knowledge, and Classroom Impact: A Research Handbook on Frameworks and Approaches. Oregon State University, USA: IGI Global.

Harriman, Cátia Luzia S. e Branch, Robert Maribe (2012) “Aligning Digital Storytelling to the TPACK Framework: A Learning Experience for Pre-Service Teachers in a Learningby-Designing Project". Congresso Brasileiro de Informática na Educação (CBIE 2012) e XVIII Workshop de Informática na Escola 2012. Disponível em: http://www.brie.org/pub/index.php/wie/article/view/2085/1852. Acesso em 28 mai. 2016.

Harris, Judith B. (2008) "TPCK in-service education: assisting experienced teachers 'planned improvisations"”. In: AACTE Committee on Innovation and Technology. Handbook of Technological Pedagogical Content Knowledge for educators. New York: Routledge. Cap. 12 , p. 251-272.

Kilbourn, Brent e Álvarez, Isabel (2008). Root-metaphors for understanding: A framework for teachers and teacher educators of information and communication technologies. In Computers \& Education, v. 50, n. 4, p. 1354-1369. Publishing Digital.

Kinchin, Ian. Avoiding technology-enhanced non-learning (2012). In British Journal of Educational Technology. v. 43, n. 2, p. E43-E48. Publishing Digital.

Koehler, Matthew e Mishra, Punya (2008) "Introducing TPACK". In: AACTE Committee on Innovation and Technology. Handbook of Technological Pedagogical Content Knowledge for educators. New York: Routledge. cap 1, p. 3-30. Publishing Digital.

Koehler, Matthew; Mishra, Punya; Yahya, Kurnia (2007). Tracing the development of teacher knowledge in a design seminar: Integrating content, pedagogy and technology. In Computers \& Education, v. 49, n. 3, p. 740-762. Publishing Digital.

Koehler, Matthew; Shin, Tae; Mishra, Punya. (2012) "How do we measure TPACK? Let me count the ways". In: Ronau, Robert N.; Rakes, Christopher R.; Niess Margaret L. Educational Technology, Teacher Knowledge, and Classroom Impact: A Research Handbook on Frameworks and Approaches. Oregon State University, USA: IGI Global. Cap. 2, p. 16-31. 
V Congresso Brasileiro de Informática na Educação (CBIE 2016)

Anais do XXII Workshop de Informática na Escola (WIE 2016)

Liu, Gi-Zen (2008) Innovating research topics in learning technology: Where are the new blue oceans? In British Journal of Educational Technology, v. 39, n. 4, p. 738-747. Publishing Digital.

Mishra, Punya e Koehler, Matthew J. (2006). Technological Pedagogical Content Knowledge: A framework for teacher knowledge. In Teachers College Record, v. 108, n.6, p. 1017-1054. Publishing Digital.

Mizukami, Maria da Graça. N. (2004). Aprendizagem da docência: algumas contribuições de L.S. Shulman. In Revista do Centro de Educação da UFSM, vol. 29, n. 02. Publishing Digital.

Nakashima, Rosária H. R. (2014) “A dialética dos conhecimentos pedagógicos dos conteúdos tecnológicos e suas contribuições para a ação docente e para o processo de aprendizagem apoiados por ambiente virtual”. 2014. 288f. Tese (Doutorado) - Faculdade de Educação, Universidade de São Paulo, São Paulo.

Nicolau, Ricardo; Araújo, Vanda e Silva, Edson (2015) “Ambientes virtuais de aprendizagem e o modelo TPACK: relato de experiência de oferta de cursos em diferentes plataformas de educação a distância" IV Congresso Brasileiro de Informática na Educação (CBIE 2015) e XXI Workshop de Informática na Escola 2015. Disponível em: http://www.brie.org/pub/index.php/wie/article/view/5046. Acesso em 28 mai. 2016.

Niess, Margaret L. (2008) "Guiding preservice teachers in developing TPCK”. In: AACTE Committee on Innovation and Technology. Handbook of Technological Pedagogical Content Knowledge for educators. New York: Routledge. Cap. 11, p. 223-250.

(2012) "Teacher knowledge for teaching with technology: a TPACK lens". In: RONAU, Robert N.; RAKES, Christopher R.; NIESS Margaret L. Educational Technology, Teacher Knowledge, and Classroom Impact: A Research Handbook on Frameworks and Approaches. Oregon State University, USA: IGI Global. Cap. 1, p. 1-15.

Peeraer, Jef e Van Petegem, Peter (2012). Measuring integration of information and communication technology in education: An item response modeling approach. In. Computers \& Education, v. 58, n. 4, p. 1247-1259. Publishing Digital.

Petko, Dominik. (2012). Teachers' pedagogical beliefs and their use of digital media in classrooms: Sharpening the focus of the 'will, skill, tool' model and integrating teachers' constructivist orientations. In Computers \& Education, v. 58, n. 4, p. 1351-1359. Publishing Digital.

Piconez, Stela C. B. e Filatro, Andrea (2009). O desenvolvimento profissional da docência na formação de professores face a utilização das tecnologias. In ETD - Educação Temática Digital, Campinas, v. 10, n. 2, p.394-427. Publishing Digital.

Prestridge, Sarah. (2012). The beliefs behind the teacher that influences their ICT practices. In Computers \& Education, v. 58, n. 1, p. 449-458. Publishing Digital.

Ronau, Robert N.; Rakes, Christopher R.; Niess Margaret L. (2012) "Educational Technology, Teacher Knowledge, and Classroom Impact: A Research Handbook on Frameworks and Approaches”. Oregon State University, USA: IGI Global.

Ronau, Robert N.; Rakes, Christopher (2012a) "A comprehensive framework for teacher knowledge (CFTK): complexity of individual aspects and their interactions". In: Ronau, Robert N.; Rakes, Christopher R.; Niess Margaret L. Educational Technology, Teacher Knowledge, and Classroom Impact: A Research Handbook on Frameworks and Approaches. Oregon State University, USA: IGI Global. Cap. 4, p. 59-102. 
V Congresso Brasileiro de Informática na Educação (CBIE 2016)

Anais do XXII Workshop de Informática na Escola (WIE 2016)

Ronau, Robert N.; Rakes, Christopher (2012a) Making the grade: reporting educational technology and teacher knowledge research. In: RONAU, Robert N.; RAKES, Christopher R.; NIESS Margaret L. Educational Technology, Teacher Knowledge, and Classroom Impact: A Research Handbook on Frameworks and Approaches. Oregon State University, USA: IGI Global, 2012b. Cap. 14, p. 323-332.

Schön, Donald. (1992) "Formar professores como profissionais reflexivos". In: Nóvoa, Antonio (Ed.) Os Professores e a sua formação. Lisboa: Dom Quixote, pp. 77-91.

Shulman, Lee S. (1986). Those who understand: Knowledge growth in teaching. In Educational Researcher, v. 15, n. 2, p. 4-14. Publishing Digital.

Shulman, Lee S. (1986). (1987). Knowledge and teaching: foundations of the New Reform. In Harvard Educational Review. v. 57, n.1, p.1-22. Publishing Digital.

Tardif, Maurice (2004) "Saberes docentes e formação profissional”. 4. ed. Petrópolis, RJ: Vozes.

Tondeur, Jo et al. (2012). Preparing pre-service teachers to integrate technology in education: A synthesis of qualitative evidence. In Computers \& Education, v. 59, n. 1, p. 134-144. Publishing Digital.

UNESCO (2008) “Padrões de competência em TIC para professores”. Paris: UNESCO.

Wilson, Carolyn et al. (2011) "Media and information literacy curriculum for teachers". Paris: UNESCO.

Yurdakul, Isil Kabakci et al. (2012) The development, validity and reliability of TPACKdeep: A technological pedagogical content knowledge scale. In Computers \& Education, v. 58, n. 3, p. 964-977. Publishing Digital. 